\title{
Proposed method for phase retardation measurements using polarimetric techniques
}

\author{
Alshaimaa W. Abdallah * \\ National Institute of Standards (NIS), Tersa St. Haram, P.O. Box: 136 Giza 12211, Egypt.
}

Global Journal of Engineering and Technology Advances, 2021, 09(02), 054-056

Publication history: Received on 05 October 2021; revised on 9 November 2021; accepted on 11 November 2021

Article DOI: https://doi.org/10.30574/gjeta.2021.9.2.0150

\begin{abstract}
Phase retardance elements such as quarter, half and full waveplates are essential elements in the most of the polarization systems. So, they should be determined precisely. Accurate determination of the phase retardation for these elements are necessary and critical due to its effect on the optical polarization measurements. In this paper we measure two-dimensional phase retardation distribution of half wave plate using four step phase shifting technique for accurate retardance measurements through the whole surface of the wave plate. By using this technique, four images are captured, which are related to different orientation of the azimuth angle of the analyzer, and mathematically analyzed to obtain the phase retardance measurements. The mean value of the phase retardance measurements is $182.57^{\circ}$ with uncertainty $0.6^{\circ}$.
\end{abstract}

Keywords: Optical retardation; Polarimetric system; Half wave plate; Four step phase shifting technique.

\section{Introduction}

The optical retardation elements are anisotropic materials like quartz, mica, polymer and calcite. These elements are used to change the polarization state of light transmitted through it with a known retardance value [1,2]. Some of these elements such as quarter, half and full wave plates are designed with certain phase retardance angle at a specific wavelength $[3,4]$. There are several well-known methods that are used for the calibration and determination of the optical phase retardance angles. Among these methods, the Senarmont compensating method which is considered as the traditional method [5-7] but it has a disadvantage that the misalignment of any of the optical components in the setup will cause error in the measurements due to that this method depend on the extinction of light using both the polarizer and analyzer to get the phase retardance measurement. Over the years, different methods have been carried out for retardance measurements by detecting the phase of young's fringes [8] or by using two compensator methods for retardation measurement [9] or by measuring the phase retardance and birefringence angles using photometric imaging polarimeter [10]. In this work, the two-dimensional distribution of the phase retardance angles of half wave plate through the whole surface are measured precisely using the four-step phase shifting technique and the mean value of the retardance angle is found in a good consistence with the results in ref [11].

\section{Experimental work}

Figure 1. Shows the four-step phase shifting technique where the system is illuminated with a laser beam. This beam is incident on a linear polarizer with its transmission angle is adjusted at $90^{\circ}$ and then the linear polarized beam is passes through a quarter waveplate with its fast axis fixed on $45^{\circ}$ and then is transmitted through the sample, second quarter wave plate and analyzer. The second quarter wave plate is rotated at a fixed azimuth angle $-45^{\circ}$ while the analyzer is rotated with different rotation angles $\alpha\left(0^{\circ}, 45^{\circ}, 90^{\circ}\right.$ and $\left.135^{\circ}\right)$.

\footnotetext{
* Corresponding author: Alshaimaa W Abdallah

National Institute of Standards (NIS), Tersa St. Haram, P.0. Box: 136 Giza 12211, Egypt. 


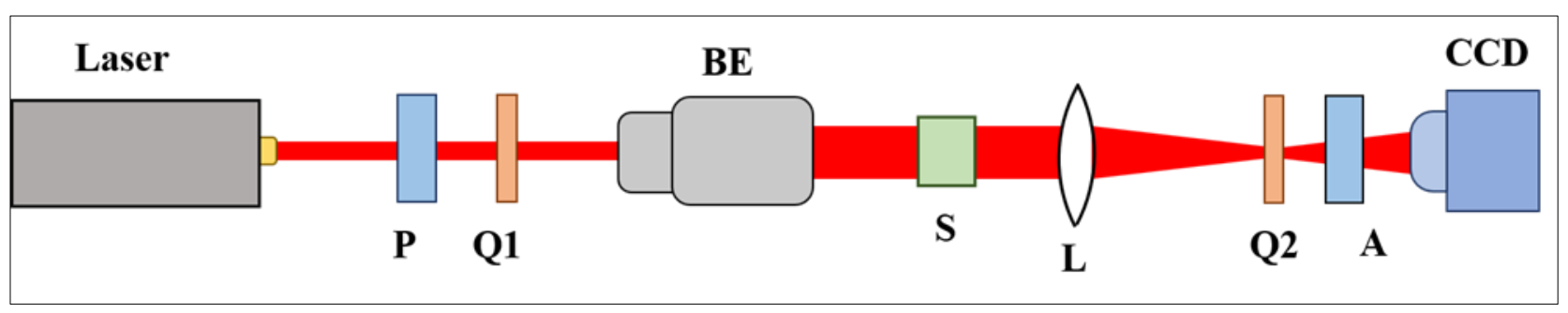

Figure 1 Four-stepping technique, where P, A are the polarizer and analyzer respectively, Q1, Q2: quarter wave plates, S: sample under test, L: convex lens and CCD: CCD camera

Four images are captured using CCD camera to measure the phase retardance angles of the half wave plate. The images are mathematically analyzed in accordance to the intensity at each pixel and the retardance angles $\delta$ can be extracted using this equation [12]:

$$
\delta=\tan ^{-1}\left(\frac{I_{4}-I_{2}}{I_{1}-I_{3}}\right)
$$

Where $I_{1}, I_{2}, I_{3}$ and $I_{4}$ are the intensity values at each rotation angle $\left(0^{\circ}, 45^{\circ}, 90^{\circ}\right.$ and $\left.135^{\circ}\right)$ respectively.

\section{Results and discussion}

In this section we show the results of the phase retardation measurements using the four-stepping method. Figure 2 represents the two-dimensional phase retardation measurements of the half wave plate at wavelength $632.9 \mathrm{~nm}$.

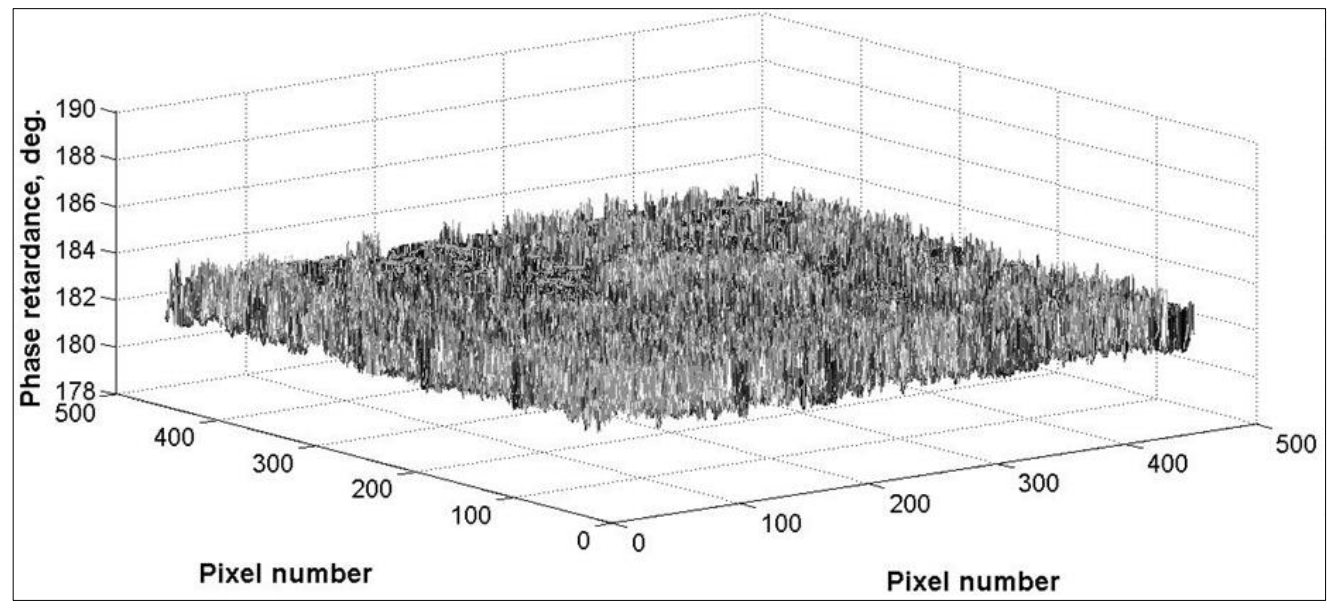

Figure 2 The two dimensional phase retardation measurements for the half wave plate using the four step polarimetric technique.

These measurements are repeated for 5 times to estimate the uncertainty of the measurements. From figure 2, the phase retardance measurements is range from $184.18^{\circ}$ to $181.47^{\circ}$ and the mean value of the retardance measurements at wavelength $632.9 \mathrm{~nm}$ is

$$
\delta=182.57^{\circ} \pm 0.6^{\circ}
$$

where the $0.6^{\circ}$ is the expanded uncertainty in measurements.

The uncertainty in phase retardance angles is estimated using the standard IS098-3 (2008) [13]. The main sources of uncertainty that contribute in the phase retardance angle measurements using the four-step method are the repeatability of measurements, the error in quarter wave plates and error in polarizers. Repeatability of the measurements has significant contribution on the estimated uncertainty and it is found to be $0.2^{\circ}$. 
The mean value of the phase retardance measurements of half wave plate at the wavelength $632.9 \mathrm{~nm}$ is found to be compatible with the results of the same plate using both the Senarmont compensating method and the polarizing interferometry method as described in ref [11].

\section{Conclusion}

The two-dimensional phase retardance measurements of half wave plate are determined precisely using four step phase shifting technique. This technique depend on the rotation of the analyzer angle at four different orientation angles and images are captured using CCD camera. The images are analyzed to get the two-dimensional mapping distribution of the phase retardance angles over the whole sample. The mean value of the phase retardance measurements of the half wave plate is found to be $182.57^{\circ}$ with uncertainty $0.6^{\circ}$ and is in a good consistence with the measurements of the half plate using Senarmont method.

\section{References}

[1] Zheng Ping Wang. Method to enhance the accuracy of the retardance measurement of quarter-wave plates, Optics and Lasers in Engineering. 2005; 43: 1226-1236.

[2] Z Wang. Novel method for measurement of retardance of a quarter-wave plate, Optics \& Laser Technology. 2004; 36: $285-290$.

[3] Jing-Fung Lin. Simultaneous measurement of optical rotation angle and retardance, Optics Communications. 2008; 281: 940-947.

[4] Jing Lin. Measurement of linear birefringence using a rotating-wave-plate Stokes polarimeter, Optik. 2010; 121: 2144-2148.

[5] NN Nagib, SA Khodier, HM Sidki. Retardation characteristics and birefringence of a multiple-order crystalline quartz plate, Opt. Laser Technol. 2003; 35: 99-103.

[6] NN Nagib. The general theory for calibrating two arbitrarily oriented phase plate, Optics and Laser Technology. 2005; 37: 151-155.

[7] NN Nagib. Polarization metrology: advanced treatment of the Senarmont compensator arrangement for calibrating phase plates, Metrologia. 2001; 38: 253-257.

[8] Emam-Ismail M. Spectral variation of the birefringence, group birefringence and retardance of a gypsum plate measured using the interference of polarized light. Opt. Laser Technol. 2009; 41: 615-621.

[9] Wang W. Determining the retardation of a wave plate by using spectroscopic method. Opt. Commun. 2001; 285: 4850-4855.

[10] A Messaadi, et al. Optical system for measuring the spectral retardance function in an extended range, Journal of the European Optical Society-Rapid Publications. 2016.

[11] AW Abdallah, M Abdelwahab. A modified method for calibration of polarimetric components using polarizing interferometry, Meas. Sci. Technol. 2021; 32: 115003.

[12] F Ratajczyk, P Kurzynowski. correct applying of the Sénarmont method with incorrect quarterwave plate, Optik. 2002; 113: 55-56.

[13] ISO 98-3, A guide to the expression of uncertainty in measurement. 2008. 